“C 2015 IEEE. Personal use of this material is permitted. Permission from IEEE must be obtained for all other uses, in any current or future media, including reprinting/republishing this material for advertising or promotional purposes, creating new collective works, for resale or redistribution to servers or lists, or reuse of any copyrighted component of this work in other works." 


\section{Analysis and Design of a Novel Linear Generator for Harvesting Oceanic Wave Energy}

\author{
Omar Farrok, M. Rafiqul Islam Sheikh and M. Rabiul Islam \\ Department of Electrical and Electronic Engineering, \\ Rajshahi University of Engineering and Technology \\ Rajshahi-6204, Bangladesh \\ Rabiulbd@hotmail.com
}

\author{
Wei $\mathrm{Xu}^{1}$, Youguang $\mathrm{Guo}^{2}$ and Jianguo $\mathrm{Zhu}^{2}$ \\ ${ }^{1}$ HUST, Wuhan, Hubei-430074, China \\ ${ }^{2}$ UTS, NSW 2007, Australia \\ Weixu@hust.edu.cn, Youguang.Guo-1@uts.edu.au \\ Jianguo.Zhu@uts.edu.au
}

\begin{abstract}
In almost every permanent magnet linear generator (PMLG), demagnetization would greatly degrade the electricity generation capability over time. This paper proposes a novel PMLG which consists of a permanent magnet excitation generator (PMEG) to supply electrical excitation to the field winding of an electromagnetic linear generator (EMLG) which functions as the main power generator. The proposed generator can reduce the demagnetization problem of the conventional PMLG. The finite element Analysis is performed by using the commercial software package ANSYS/ANSOFT for designing the proposed PMLG, and the genetic algorithm has been used to find the optimal pole size, pole pitch, air gap length and load variation to maximize the output power. Special m-shaped stator core is designed for the PMEG to minimize the leakage flux and cogging force. The voltage, current, power, magnetic flux density, force components and applied force are also analyzed and discussed.
\end{abstract}

Keywords - EMLG; excitation generator; m-core.

\section{INTRODUCTION}

Because of the global energy crisis, scientists and engineers are working hard to solve the problem by utilizing renewable energy sources [1], [2]. Oceanic wave has high power density compared to wind or solar energy and it is available, predictable and environment friendly [3]. Renewable power generation, however, fluctuates most of the time, depending upon the environmental conditions which can vary on the time scales from seconds to days. Because of this, there are significant challenges for power grids to manage the stability, security and reliability while smoothing the generated power and thus make the best use of renewable energy sources [4]. At present, the major linear generators used for wave energy extraction are permanent magnet (PM) linear synchronous machines that usually have PMs on the translator [5], [6]. The traditional linear generators have the disadvantages of complex translator structure and unexpected translator temperature rise, which might cause irreversible demagnetization of magnets and mechanical damage [7].

This paper proposes a novel linear generator which consists of a PM excitation generator (PMEG) and an electromagnetic linear generator (EMLG). NdFeB PMs are mounted on the translator and special $\mathrm{m}$-shaped stator cores are employed to reduce the core losses so as to overcome demagnetization problem by using electromagnets and have longer lifetime. Unlike the conventional permanent magnet linear generator
(PMLG) which has fixed flux and thus the generated electrical power fluctuates with the wave motion, the proposed EMLG can readily regulate the output power by controlling the field excitation.

\section{ThE PROPOSED SySTEM}

Fig.1 illustrates the excitation system or PMLG for the proposed EMLG, where the three phase AC voltages produced by the PMLG, $E_{1}, E_{2}$ and $E_{3}$, are rectified by a diode bridge into DC voltage to supply the field windings of four EMLG units. In Fig.1, the three phase voltages, $E_{a}, E_{b}$, and $E_{c}$, of one EMLG unit are shown, where $R_{f}, R_{a}$ and $R_{L}$ are the field, armature and load resistances, respectively.

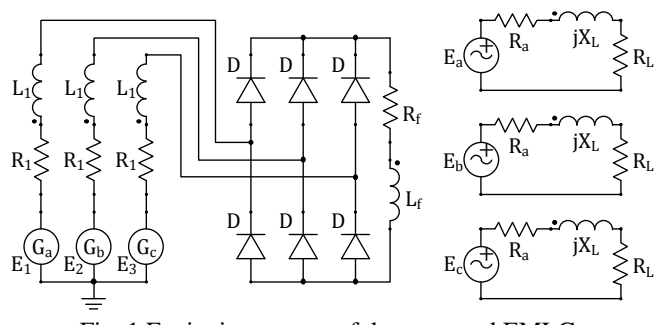

Fig. 1 Excitation system of the proposed EMLG.

Fig.2 illustrates the structure of proposed PMLG, where blue and brown colors represent the PMs with opposite magnetization directions to form the north $(\mathrm{N})$ and south $(\mathrm{S})$ poles in the translator. Fig.3 plots the finite element meshes.

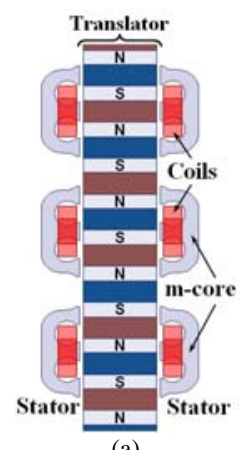

(a)

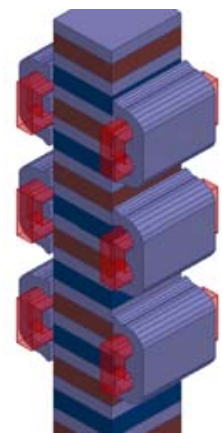

(b)

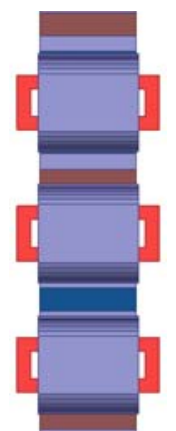

(c)
Fig. 2 Structure of the proposed PMEG, (a) front view, (b) isometric view, and (c) right side view. 


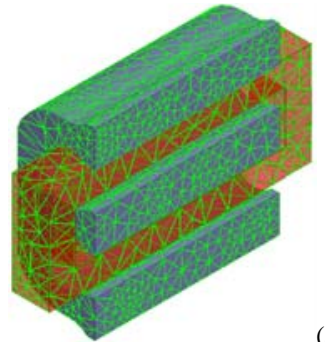

(a)

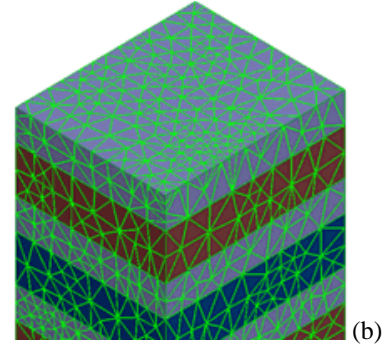

Fig. 3 Mesh plot of the proposed PMEG, (a) m-core with coil, and (b) translator.

Fig.4 plots the proposed EMLG construction, flux density which varies with time and dimensions.

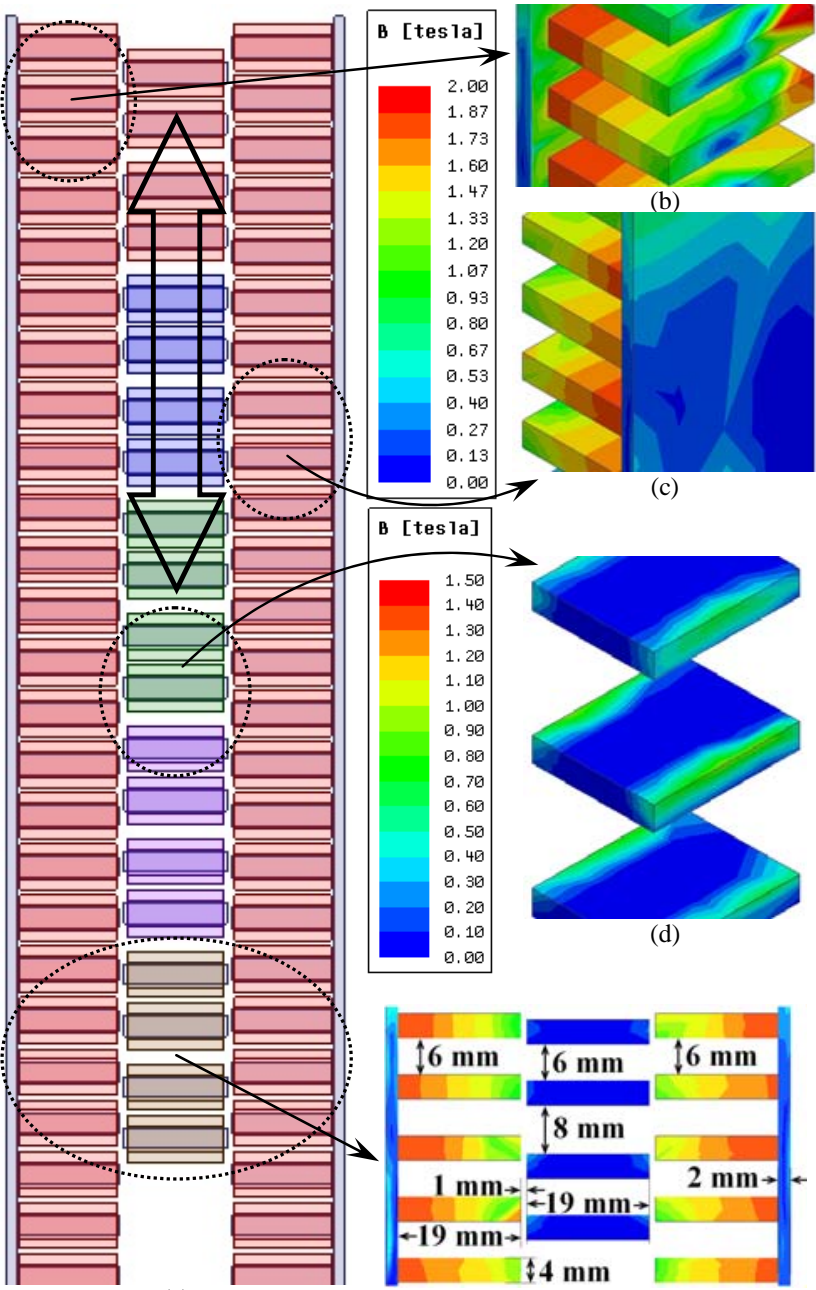

(a)

(e)

Fig. 4 Design of the proposed EMLG: (a) the front view, (b) flux density of stator core of front side, (c) rear side, (d) translator core, and (e) dimension.

\section{SIMULATION RESULTS}

In the simulation, the translator velocity is set to $1 \mathrm{~m} / \mathrm{s}$. All of the main winding coils have 200 turns. The output voltage and current of the proposed PMLG is shown in Fig 5. The total rms power output of PMLG and EMLG, different force components, and applied force are shown in Fig 6. The applied force works in the upward direction which is noted as Force_Z. The negative value indicates that the force is applied to the translator. Force_X and Force_Y are force components, working in the horizontal plane due to electromagnetic effects between the stator and translator.

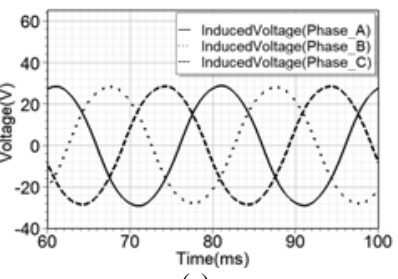

(a)

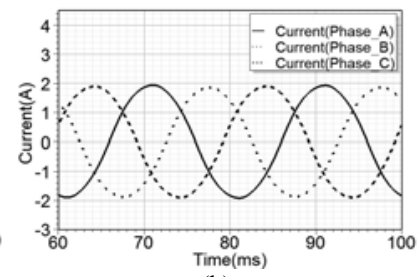

(b)
Fig. 5 Output waveforms of the proposed EG: (a) voltage, and (b) current.

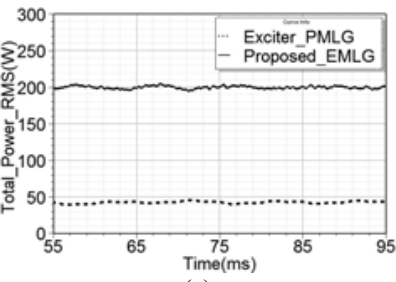

(a)

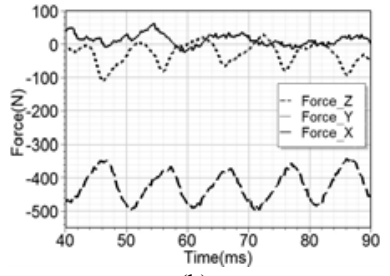

(b)
Fig. 6 (a) The output power of PMLG and EMLG, and (b) the applied force components.

\section{CONCLUSIONS}

The simulation results have shown that the proposed LG can generate electrical power effectively as in the conventional PMLG, but it does not have the problem of overheating and demagnetization. By controlling the field excitation, the output power of EMLG can be regulated such that it does not vary with the motion of waves. The simulation results have shown clearly that the force ripples and the cogging forces are successfully minimized by by pole shifting and the use of special m-cores.

\section{REFERENCES}

[1] M. R. Islam, Y. G. Guo, J. G. Zhu, H. Y. Lu, and J. X. Jin, "Highfrequency magnetic-link medium-voltage converter for superconducting generator-base high power density wind generation systems," IEEE Tran. Appl. Superconduct., vol. 24, no. 5, Art. 5202605, October 2014

[2] M. R. Islam, G. Lei, Y. G. Guo, and J. G. Zhu, “Optimal design of highfrequency magnetic-links for power converters used in grid connected renewable energy systems,” IEEE Trans. Magn., vol. 50, no. 11, Art. 2006204, November 2014.

[3] J. Prudell, et al., "A permanent-magnet tubular linear generator for ocean wave energy conversion,” IEEE Trans. Ind. Appl., vol. 46, no. 6 , pp. 2392, 2400, Nov. -December 2010.

[4] H. Zhang, et al., "Design and simulation of SMES system using YBCO tapes for direct drive wave energy converters," IEEE Tran. Appl. Superconduct., vol. 23, no. 3, pp. Art. 5700704, June 2013.

[5] N. M. Kimoulakis, A. G. Kladas, and J. A. Tegopoulos, "Power generation optimization from sea waves by using a permanent magnet linear generator drive," IEEE Trans. Magn., vol. 44, no. 6, pp. 1530 1533, June 2008

[6] L. Huang, et al., "Winding configuration and performance investigations of a tubular superconducting flux-switching linear generator," IEEE Tran. Appl. Superconduct., vol. 25, no.3, pp. Art. 5700704, June 2015.

[7] L. Huang, H. Yu, M. Hu, J. Zhao, and Z. Cheng, "A novel fluxswitching permanent-magnet linear generator for wave energy extraction 
application,” IEEE Trans. Magn., vol. 47, no. 5, pp. 1034-1037, May 2011. 